\title{
Magneto-Impedance Biosensor With Enhanced Sensitivity for Highly Sensitive Detection of Nanomag-D Beads
}

\author{
Jagannath Devkota, Alejandro Ruiz, Pritish Mukherjee, Hariharan Srikanth, and Manh-Huong Phan \\ Department of Physics, University of South Florida, Tampa, FL 33620 USA
}

\begin{abstract}
A magnetoimpedance (MI) biosensor based on Co-based amorphous ribbon was designed and tested to detect functionalized Nanomag-D magnetic beads. While previous studies were focused mainly on exploring the MI change for biosensing, we show that the sensitivity of the biosensor can be enhanced when the change in ac magnetoresistance (MR) or magnetoreactance (MX) is used. The frequency at which the sensitivity of the sensor is optimized can be tuned. This is of potential interest in developing functional biosensors with improved sensitivity and tunable frequency.
\end{abstract}

Index Terms-Magnetic biosensors, magnetoimpedance (MI), magnetoreactance (MX), magnetoresistance (MR).

\section{INTRODUCTION}

M AGNETIC biosensors are of increasing research interest for their high potential to target biomolecules through the detection of functionalized magnetic beads [1], [2]. The important requirements of a biosensor regarding the detection of magnetic beads include high sensitivity, low power consumption, quick response, reliability, environment-friendly operation, and low cost. Magnetic sensors based on various principles, such as giant magnetoresistance (GMR) [3], [4] and superconducting quantum interference device (SQUID) [5], have been developed to detect magnetic beads. However, GMR sensors possess limited sensitivity and SQUID sensors require extremely low temperatures for operation. Recently, alternative biosensors based on the giant magnetoimpedance (GMI) effect of soft ferromagnetic materials have been proposed for sensitive detection of magnetic biomarkers [6]-[9]. They are highly sensitive to the applied field, low power consuming, and operational at room temperature. The GMI effect is a large change in the complex impedance $Z=R+\mathrm{j} X$ (where $R$ and $X$ are the resistance and reactance, respectively) of a soft ferromagnetic conductor subject to an external dc magnetic field [10].

GMI-based biosensors have been successfully employed to study the relative change in the impedance of amorphous ribbons [6], [8] and microwires [7] from the presence of added magnetic particles, such as commercial dynabeads [6], estapor beads [7], and superparamagnetic iron oxide $\left(\mathrm{Fe}_{3} \mathrm{O}_{4}\right)$ [8]. However, targeting a very low concentration of a biological sample, such as cancer cells that have taken up magnetic nanoparticles [11], [12], requires more sensitivity for nanoparticle detection. Therefore, the increment of sensitivity and functional character of a sensor are of current focus in developing a new generation of diagnostics systems.

In this paper, we report on a systematic study of GMI detection of functional magnetic beads, Nanomag-D, using a Co-based amorphous ribbon. Instead of limiting the focus exclusively to the impedance change for biosensing, as in previous studies [6]-[9], [11], [12], we show that the sensitivity of a GMI-based biosensor can be enhanced when a change in ac resistance and/or reactance is used. The frequency at which the highest sensitivity of the sensor is achieved can be tuned as well. Treating the surface of a ribbon with an appropriate concentration of $\mathrm{HNO}_{3}$ acid is also shown to improve the sensitivity of detection of a ribbon-based GMI biosensor.

\section{EXPERIMENT}

Functionalized Nanomag-D beads (diameter, $\sim 250 \mathrm{~nm}$ ) suspended in water with the original concentration of $10 \mathrm{mg} / \mathrm{ml}$ were purchased from Mircomod Partikeltechnologie $\mathrm{GmbH}$, Germany. These beads are $\mathrm{NH}_{2}$-coated composites of iron oxide nanoparticles and dextran and have potential medical applications [13], [14]. The sensor prototype has dimensions of $16 \mathrm{~mm} \times 2 \mathrm{~mm} \times 0.015 \mathrm{~mm}$ and was designed using a Co-based amorphous ribbon $\left(\mathrm{Co}_{65} \mathrm{Fe}_{4} \mathrm{Ni}_{2} \mathrm{Si}_{15} \mathrm{~B}_{14}\right)$ prepared by a rapid quenching technique. The impedance was measured using an HP4192A impedance analyzer over a length of $1 \mathrm{~cm}$ by the four point measurement technique with a constant current of $5 \mathrm{~mA}$ flowing along the length of the ribbon in a frequency range of $0.1-13 \mathrm{MHz}$ in the presence of axial dc magnetic field of up to \pm 120 Oe. The impedance was first measured for a plain ribbon (P), and then with $30 \mu \mathrm{l}$ of a Nanomag-D suspension (Nmag, $100 \mu \mathrm{g} / \mathrm{ml}$ ) drop-cast on it for comparison. To improve the sensitivity of particle detection, the surface of the ribbon was also treated with $5 \mu \mathrm{l}$ of dilute $(\sim 4.5$ vol- $\%) \mathrm{HNO}_{3}$ for $24 \mathrm{hrs}$ and washed with distilled water. The impedance was measured again for the acid-treated ribbon (A), with $5 \mu \mathrm{l}$ of water, and Nanomag-D suspension, separately. The magnetoresistance (MR), magnetoreactance (MX), and magnetoimpedance (MI) ratios were respectively defined as

$$
\begin{aligned}
& \frac{\Delta R}{R}=\frac{R(H)-R\left(H_{\max }\right)}{R\left(H_{\max }\right)} \times 100 \% \\
& \frac{\Delta X}{X}=\frac{X(H)-X\left(H_{\max }\right)}{X\left(H_{\max }\right)} \times 100 \% \\
& \frac{\Delta Z}{Z}=\frac{Z(H)-Z\left(H_{\max }\right)}{Z\left(H_{\max }\right)} \times 100 \%
\end{aligned}
$$



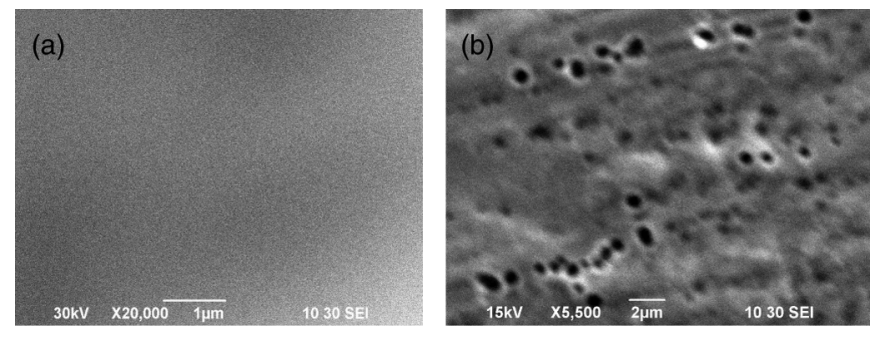

Fig. 1. SEM image of Co-based (a) plain and (b) acid-treated ribbons.

The changes in MR, MX, and MI ratios due to the presence of the beads were obtained as

$$
\Delta \eta_{\xi}=[\xi]_{\text {max }, \text { bead }}-[\xi]_{\text {max, water }}
$$

where $[\xi]_{\max }$ with $\xi=\Delta R / R, \Delta X / X$, and $\Delta Z / Z$ are the maximum values of MR, MX, and MI ratios in (1), (2), and (3) respectively.

\section{RESUlTS AND DISCUSSION}

We first investigated the influence of acid on the surface morphology of a plain ribbon using scanning electron microscopy (SEM). Fig. 1 shows SEM images of the surface morphology for the plain ribbon (a) and acid-treated ribbon (b). From the figure, it is observed that the surface roughness increased when the ribbon was treated with diluted $\mathrm{HNO}_{3}$. While acid etch is known to clean the surface and remove impurities, the corrosive nature also results in etch pits as observed in Fig. 1(b).

Fig. 2 shows the dc field dependence of MI ratio at $1.5 \mathrm{MHz}$ for the plain ribbon $(\mathrm{P})$ and the acid-treated ribbon $(\mathrm{A})$, compared with the Nanomag-D-coated ribbon (Nmag-100 $\mu \mathrm{g} / \mathrm{ml}$ ). It can be seen that the GMI ratio for all samples varied in a similar fashion with the applied field. As expected, the presence of Nanomag-D beads on the ribbon surface resulted in the increase of the GMI ratio in both untreated and acid-treated ribbon samples. It is interesting to note in Fig. 2 that while the acid treatment reduced the GMI ratio of the plain ribbon, the difference in the GMI ratio observed for the acid treated ribbon with and without Nanomag-D beads is about three times larger than that observed for the untreated ribbon with and without Nanomag-D beads. This indicates that treating the surface of a ribbon with an appropriate concentration of acid can be an effective way for improving the detection of a ribbon-based GMI biosensor. In a recent study, we have shown that stray fields arising from rough surfaces cause a considerable reduction in GMI and reducing the surface roughness of a ribbon by coating it with a thin magnetic metal layer enhances the GMI ratio [15]. So, a reduction in the GMI ratio of the ribbon on treating its surface with an acid due to increased surface roughness is expected in the present case. As compared to the case of the smooth surface, however, the presence of magnetic nanoparticles on the rough surface of the ribbon results in a larger change in GMI. This is because the GMI signal of the rough surface is more sensitive to stray fields coming from Nanomag-D magnetic beads. The presence of etch pits on the surface of the acid-treated ribbon [see Fig. 1(b)] seems to play an important role in improving

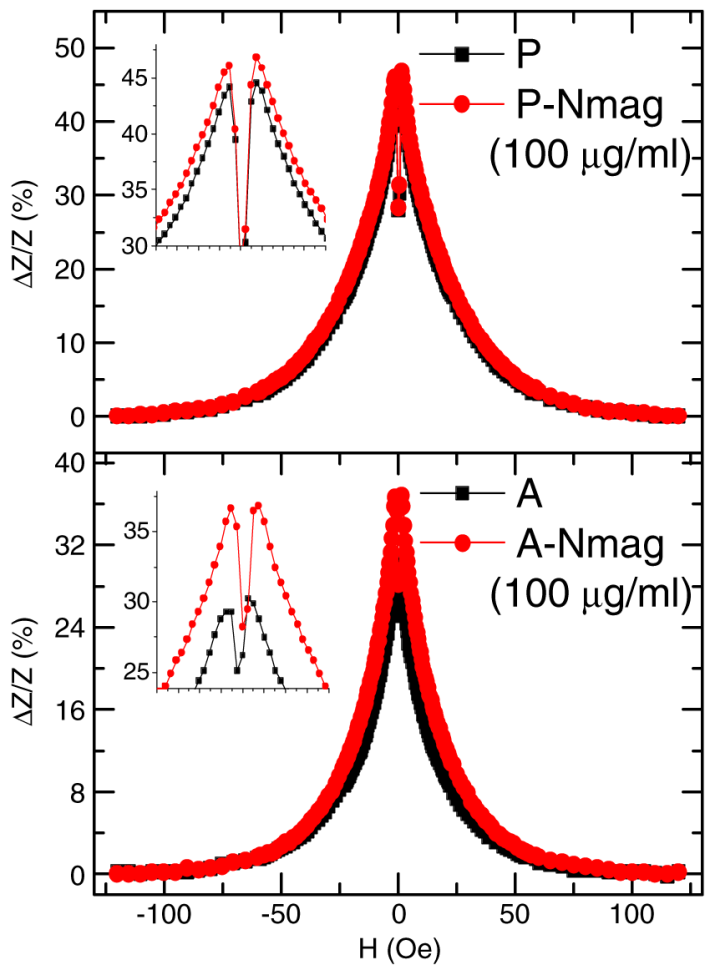

Fig. 2. Magnetic field dependence of magneto-impedance for samples (a) $\mathrm{P}$ and P-Nmag $(100 \mu \mathrm{g} / \mathrm{ml})$ and (b) A and A-Nmag $(100 \mu \mathrm{g} / \mathrm{ml})$.

the sensitivity of particle detection [see Fig. 2(b)]. The physical stability of the magnetic beads and their distance from the sensing element are also important factors for the detection of a stray field [16], [17]. Keeping this in mind, we have chosen the acid-treated sample for studying the magnetic field and frequency dependences of MR, MX, and MI with and without the presence of Nanomag-D beads on the ribbon surface.

Fig. 3 shows the magnetic field dependence of MR, MX, and MI ratios at the selected frequencies of $1 \mathrm{MHz}$ and $10 \mathrm{MHz}$ for the acid-treated ribbon (A), with water (A-W), and with 250 $\mu \mathrm{g} / \mathrm{ml}$ Nanomag-D (A-Nmag- $250 \mu \mathrm{g} / \mathrm{ml}$ ). There was no significant change in the MR, MX, and MI ratios of the ribbon when $5 \mu \mathrm{l}$ distilled water was drop-casted on its surface. However, there was a clear increase in MR, MX, and MI ratios in the presence of equal volume of Nanomag-D suspension. This increase in the GMI ratio of the ribbon is due to the disturbances on the applied dc field and transverse ac field by the fringe field of the magnetic particles [4], [6].

To better illustrate the relative change in GMI due to the presence of Nanomag-D beads, we display in Fig. 4(a)-(c), the frequency dependence of the maximum MR, MX, and MI ratios for the acid-treated (A), with water (A-W), and with Nanomag-D suspension (A-Nmag-250 $\mu \mathrm{g} / \mathrm{ml}$ ). It is observed that the MR ratio of all samples increases with frequency [Fig. 4(a)]. The MX ratio has a maximum at lowest frequency [see Fig. 4(b)] and decreases as the frequency increases. Finally, the MI ratio [see Fig. 4(c)] increases with frequency, reaches a maximum and decreases slowly. This feature implies that the GMI effect is inductive at low frequencies and resistive at high frequencies, however, both of them contribute at intermediate frequencies, where the peak of the MI lies. The MR, MX, and MI ratios for 

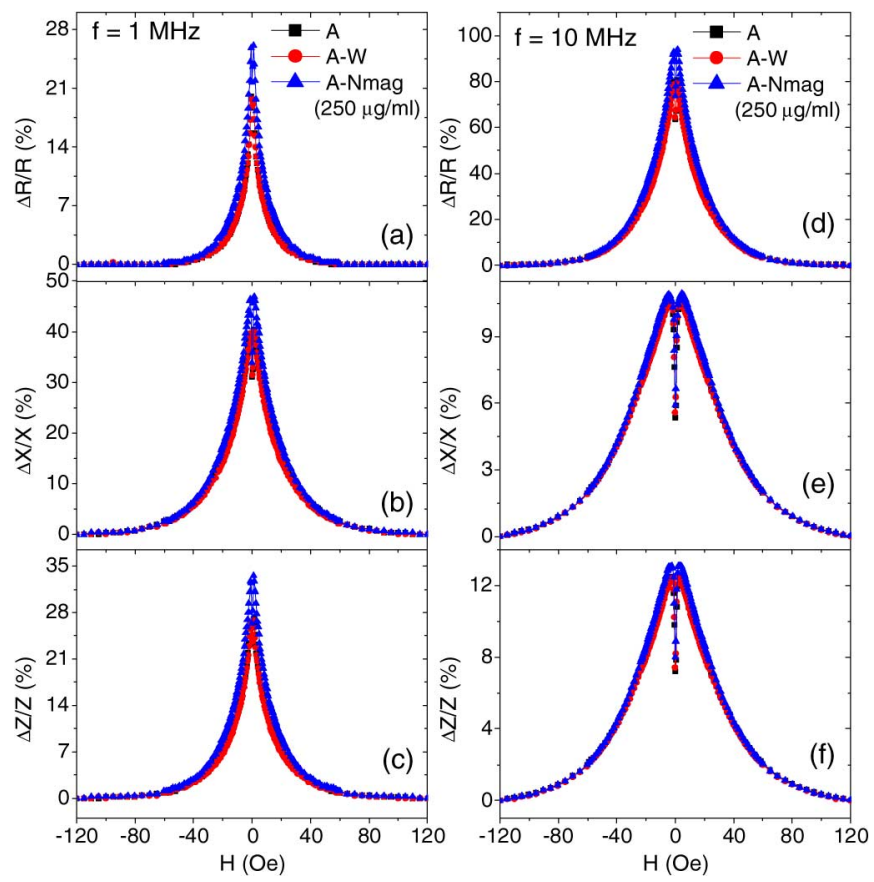

Fig. 3. Magnetic field dependence of (a), (d) magneto-resistance, (b), (e) magneto-reactance, and (c), (f) magneto-impedance at frequencies of 1 and $10 \mathrm{MHz}$ for samples A, A-W, and A-Nmag $(250 \mu \mathrm{g} / \mathrm{ml})$.

the samples $\mathrm{A}$ and $\mathrm{A}-\mathrm{W}$ are almost equal across the whole investigated frequency range of $0.1-13 \mathrm{MHz}$, indicating that there is no effect of water on them. However, these ratios are found to increase significantly for the case of A-Nmag. This indicates that the presence of the magnetic beads can be identified using the GMI-based sensor. To assess the sensitivity of the sensor, we have calculated the difference in maximum of MR, MX, and MI $\left(\Delta \eta_{\xi}\right)$ for the Nanomag-D suspension and water; these results are displayed in Fig. 4(d). From this figure, the $\Delta \eta_{\xi}$ s for MR, MX, and MI show different frequency-dependent behaviors. The highest values of $\Delta \eta_{\xi}$ observed for MR, MX, and MI due to the presence of Nanomag-D beads are $16 \%, 23 \%$, and $7 \%$ at $\mathrm{f}=10,0.1$, and $0.7 \mathrm{MHz}$, respectively. It is clear that the sensitivity of the sensor is enhanced by two or three times as the change in MR or MX is used. The sensitivity of the MR-based sensor is found to increase with frequency up to $10 \mathrm{MHz}$ and it is about ten times higher than those of the MX- and MI-based sensors at $f \sim 10 \mathrm{MHz}$. These results indicate that it is possible to use the combined measurements and analysis of MR, MX, and MI for making a functional biosensor with enhanced sensitivity and tunable working frequency.

To explain the origin of the observed effects, we recall that the impedance of the ribbon is a function of the driving current frequency $(f)$ and external dc magnetic field $\left(H_{d c}\right)$ through magnetic permeability $\left(\mu_{T}\right)$ and skin depth $\left(\delta_{m}\right)$ given by $\delta_{m}=\sqrt{\rho / \pi \mu_{T} f}$, where $\rho$ is the resistivity of the material [9]. The GMI effect is often observed at high frequencies $(f>1 \mathrm{MHz})$, where the skin effect is significant enough to confine the ac current to a sheath close to the surface of the ribbon. This makes GMI very sensitive to the surface conditions of the ribbon. When the magnetic beads are present on
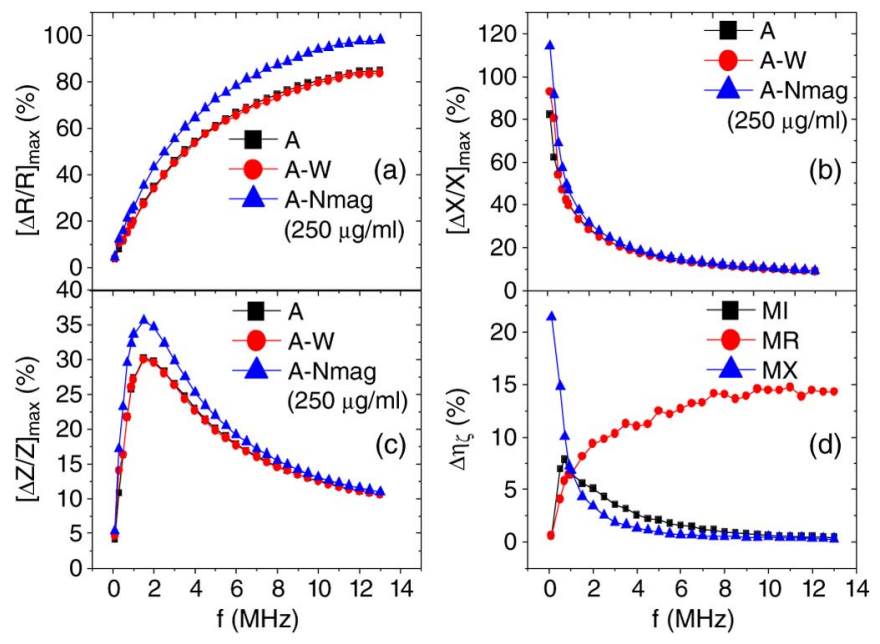

Fig. 4. Frequency dependence of maximum (a) magneto-resistance, (b) magneto-reactance, (c) magneto-impedance for samples A, A-W, and A-Nmag $(250 \mu \mathrm{g} / \mathrm{ml})$, and (d) the difference in MR, MX, and MI between the A-W and A-Nmag $(250 \mu \mathrm{g} / \mathrm{ml})$.

the surface of the ribbon in a transverse ac magnetic field and a longitudinally-applied dc magnetic field, they can be magnetized in either direction [18], [19]. The stray field produced due to magnetization interacts with the ac field and applied dc field resulting in the modification in transverse permeability. In our case, it can be assumed that the majority of the beads magnetized in the transverse direction resulting in increased transverse permeability, and hence, enhanced MR, MX, and MI ratios. This could be due to the coupling of the stray field of the nanoparticles with the transverse ac field that increases the transverse permeability thereby causing high change in the impedance.

\section{CONCLUSION}

We performed a systematic study on the detection of Nanomag-D magnetic beads using an amorphous ribbon-based magnetic biosensor. The changes of the real and imaginary components of the magneto-impedance are shown to be promising for highly sensitive detection of magnetic nanoparticles in biological systems where one has to deal with very small sample size. The sensitivity of the biosensor can be enhanced on treating the surface of the ribbon with an appropriate concentration of $\mathrm{HNO}_{3}$.

\section{ACKNOWLEDGMENT}

The work at USF was supported by the Florida Cluster for Advanced Smart Sensor Technologies (FCASST) and by USAMRMC through grant numbers W81XWH-07-1-0708 and W81XWH1020101/3349. Metglas/Hitachi Metals America was acknowledged for providing Cobalt based MetglasVR Inc. $2705 \mathrm{M}$ ribbons.

\section{REFERENCES}

[1] A. P. F. Turner, "Biosensors—Sense and sensitivity," Science, vol. 290, pp. 1315-1317, 2000 
[2] C. R. Tamanaha, S. P. Mulvaney, J. C. Rife, and L. J. Whitman, "Magnetic labeling, detection, and system integration," Biosens. Bioelectron., vol. 24, pp. 1-13, 2008.

[3] D. R. Baselt, G. U. Lee, M. Natesan, S. W. Metzer, P. E. Sheehan, and R. Colton, "A biosensor based on magnetoresistance technology," Biosens. Bioelectron., vol. 13, pp. 731-739, 1998.

[4] J. C. Rife, M. M. Miller, P. E. Sheehan, C. R. Tamanaha, M. Tondra, and L. J. Whitman, "Design and performance of GMR sensors for the detection of magnetic microbeads in biosensors," Sens. Actuators A, vol. 107, pp. 209-218, 2003.

[5] D. Drung, C. Assmann, J. Beyer, A. Kirste, M. Peters, F. Ruede, and T. Schurig, "Highly sensitive and easy-to-use SQUID sensors," IEEE Trans. Appl. Supercond., vol. 17, pp. 699-704, 2007.

[6] G. V. Kurlyandskaya and V. Levit, "Magnetic Dynabeads detection by sensitive element based on giant magnetoimpedance," Biosens. Bioelectron., vol. 20, pp. 1611-1616, 2005.

[7] H. Chiriac, D. D. Herea, and S. Corodeanu, "Microwire array for giant magneto-impedance detection of magnetic particles for biosensor prototype," J. Magn. Magn. Mater., vol. 311, pp. 425-428, 2007.

[8] A. Kumar, S. Mohapatra, V. Fal-Miyar, A. Cerdeira, J. A. Garcia, H. Srikanth, J. Gass, and G. V. Kurlyandskaya, "Magnetoimpedance biosensor for $\mathrm{Fe}_{3} \mathrm{O}_{4}$ nanoparticles intracellular uptake evaluation," Appl. Phys. Lett., vol. 91, p. 143902, 2007.

[9] G. V. Kurlyandskaya, "Giant magnetoimpedance for biosensing: Advantages and shortcomings," J. Magn. Magn. Mater., vol. 321, pp. 659-662, 2009.

[10] M. H. Phan and H. X. Peng, "Giant magnetoimpedance materials: Fundamentals and applications," Prog. Mater. Sci., vol. 53, pp. 323-420, 2008.

[11] H. Yang, L. Chen, C. Lei, J. Zhang, D. Li, Z. M. Zhou, C. C. Bao, H. Y. Hu, X. Chen, F. Cui, S. X. Zhang, Y. Zhou, and D. X. Cui, "Giant magnetoimpedance-based microchannel system for quick and parallel genotyping of human papilloma virus type 16/18," Appl. Phys. Lett., vol. 97, p. 043702, 2010.
[12] L. Chen, C. C. Bao, H. Yang, D. Li, C. Lei, T. Wang, H. Y. Hu, M. He, Y. Zhou, and D. X. Cui, "A prototype of giant magnetoimpedancebased biosensing system for targated detection of gastric cancer cells," Biosens. Bioelectron., vol. 26, pp. 3246-3253, 2011.

[13] S. J. DeNardo, G. L. DeNardo, L. A. Miers, A. Natarajan, A. R. Foreman, C. Gruenttner, G. N. Adamson, and R. Ivkov, "Development of tumor targeting bioprobes ( ${ }^{111}$ In-chimeric L6 monoclonal antibody nanoparticles) for alternating magnetic field cancer therapy," Clin. Cancer Res., vol. 11, pp. 7087s-7092s, 2005.

[14] H. Khursid, S. H. Kim, M. J. Bonder, L. Colak, B. Ali, S. I. Shah, K. L. Klick, and G. C. Hadjipanayis, "Development of heparin-coated magnetic nanoparticles for targeted drug delivery applications," J. Appl. Phys., vol. 105, p. 07B308, 2009.

[15] N. Laurita, A. Chaturvedi, C. Bauer, P. Jayathilaka, A. Leary, C. Miller, M. H. Phan, M. E. McHenry, and H. Srikanth, "Enhanced GMI effect and field sensitivity in Co-coated soft ferromagnetic amorphous ribbons," J. Appl. Phys., vol. 109, p. 07C706, 2011.

[16] B. M. de Boer, J. A. H. M. Kahlman, T. P. G. H. Jansen, H. Duric, and J. Veen, "An integrated and sensitive detection platform for magnetoresistive biosensors," Biosens. Bioelectron., vol. 22, pp. 2366-2370, 2007.

[17] M. Donolato, M. Gobbi, P. Vavassori, M. Leone, M. Cantoni, V. Metlushko, B. Ilic, M. Xhang, S. X. Wang, and R. Bertacco, "Nanosized corners for trapping and detecting magnetic nanoparticles," Nanotechnology, vol. 20, pp. 385501-385506, 2009.

[18] C. P. Gooneratne, C. Liang, A. Useinov, J. Kosel, and I. Giouroudi, "A half-ring GMR sensor for detection of magnetic beads immobilized on a circular micro-trap," in Proc. 2011 5th Int. Conf. Sens. Technol., pp. $106-111$.

[19] M. M. Miller, P. E. Sheehan, R. L. Edelstein, C. R. Tamanaha, L. Zhong, S. Bounnak, L. J. Whitman, and R. J. Colton, "A DNA array sensor utilizing magnetic microbeads and magnetoelectronic detection," J. Magn. Magn. Mater., vol. 225, pp. 138-144, 2001. 